\title{
Self-healing Model Construction and Simulation of Power SDH Transmission Network
}

\author{
Jiye Wang, Geng Zhang, Yanan Wang, Xiangzhou Chen and Yang Wang \\ China Electric Power Research Institute, No.15, QingheXiaoying, East Road, Haidian District, Beijing, China
}

\begin{abstract}
With the rapid development of Synchronous Digital Hierarchy (SDH) technology, it has been increasingly used in power communication networks. The power communication network has high requirements on the reliability of data transmission, and the self-healing ring has the advantages of automatic fault recovery, high reliability, flexible networking, and simple implementation, which has become an indispensable part of the construction of power communication SDH networks. How to effectively evaluate the self-healing capabilities of SDH and improve the performance indicators of the transmitted services has become an urgent problem to be solved. In the actual operation of the network, the self-healing ability of SDH cannot be accurately and effectively evaluated. Therefore, based on the Exata simulation platform, this article models the SDH transmission network equipment, network, services and other resources as well as the self-healing behaviors, and realizes the whole process simulation of self-healing in the SDH transmission network. Finally, the availability of access network and backbone network are compared and evaluated in a certain area, which verifies that the channel protection function of SDH self-healing ring can effectively improve the security and reliability of the network.
\end{abstract}

Keywords-SDH transmission network; self-healing model; simulation implementation

\section{INTRODUCTION}

Recently, with the increasingly widespread application of SDH transmission network in power communication networks, it becomes more and more important to improve the transmission networks survivability. Therefore, the self-healing function evaluation of the SDH network has attracted more and more attentions[1-4]. The actual measurement and evaluation of self-healing capabilities in SDH power communication networks are prone to several problems, such as the impact of larger network security and the lack of available real-world network support, etc. Therefore, combined with the actual selfhealing process of power communication networks, simulation techniques and methods are used to analyze the self-healing capabilities of SDH networks and their impact on service performance, which is necessary for the actual power grid security and power system performance evaluation.

At present, some researchers have studied the SDH optical network self-healing protection and network simulation models, such as Li Yang in Ningxia Electric Power Company, studied the effect of SDH optical fiber self-healing protection mode on the power communication system[5], and put forward a suitable network protection scheme. Wang Hua in Qinghai Electric Power, studied the number of transmission nodes, delay and protection method of SDH fiber ring network. Based on SDH (Synchronous Digital Hierarchy) self-healing mechanism, this paper analyzed the protection mechanism of electric power communication network, and introduced the defects of existing self-healing strategies, come to a conclusion that the multiplexing protection business should disable SNCP(Sub Network Connection Protection)[6]. Bai Jingru in Beijing University of Posts and Telecommunications, studied the improved method of SDH self-healing ring, through analyzing the protection switching principle, switching time and capacity in different situations, and proposed various application suggestions for the structure of different network topologies of SDH self-healing rings[7]. Most of the above studies have studied the mechanism, optimization and application of SDH self-healing rings. However, there has been no research on the self-healing ability assessment of SDH optical networks, and it is impossible to evaluate the capabilities of the designed SDH self-healing rings.

In addition, existing data network simulation platforms, such as OPNET and NS2, lack support for SDH optical network simulation[8]. Calvet J.T and Girault C. designed and developed the SDH synchronization network programming simulation platform, simulated the impact of different synchronization configurations[9]. Schwartz S. H. and Zager D. designed a discrete event based on telecommunications network simulation platform to simulate the failure process of various types of networks including SDH networks[10]. Ruepp S. et al proposed a self-healing simulation model of SDH transmission network based on database, which focused on the occurrence of simulated failures and service recovery processes[11]. Li Jianhua and others of Communication Engineering College of PLA Technical University in China developed a SDH optical Terminal Equipment simulator based on client-server model, simulated SDH network management system configuration, fault management, performance management and billing management and other functions. However, this system is mainly aimed at the training of network management operations. It is used for SDH students to master the network configuration and operation functions, and lacks deep simulations of the SDH protocol system, technology system, and equipment[12]. The above studies mostly simulated the network topology through the bottom-up modeling at the process layer, node layer and network layer, and cannot simulate the SDH network from the service perspective. In addition, in the existing network simulation model research, the network resource models and alarm models are mainly studied. The SDH optical network self-recovery simulation research has not been carried out, it is impossible to 
simulate the behavior model of network resources and to accurately simulate the self-healing process of the protection switching behavior of the service. Therefore, based on the exata simulation platform, this paper implements a SDH selfhealing process simulation including resource simulation and behavior simulation. The resources of equipment, network and application in SDH network are also involved. Additionally, the behaviors and state changes of the SDH equipments when a fault occurs, and the entire process of the self-healing function in the power SDH transmission network are realized in this paper.

\section{SDH SELF-HEALING SimUlation}

\section{A. SDH Resources Simulation}

In this paper, SDH optical transmission network resources are divided into three levels: device layer, network layer and application layer. The service layer modeling is mainly physical network modeling, such as physical devices and physical circuits; the network layer modeling is about protocol modeling, including network elements, logic processes and link logic processes; the service layer modeling is mainly about application network, including transport applications.

\section{1) Device layer modeling}

The mainly basic device layer elements in SDH optical transmission network are shown in the Figure 1, which are composed with transmitter, terminal multiplexer(TM), add/drop multiplexer(ADM), regenerator(REG), digital cross connect(DXC) and receiver.

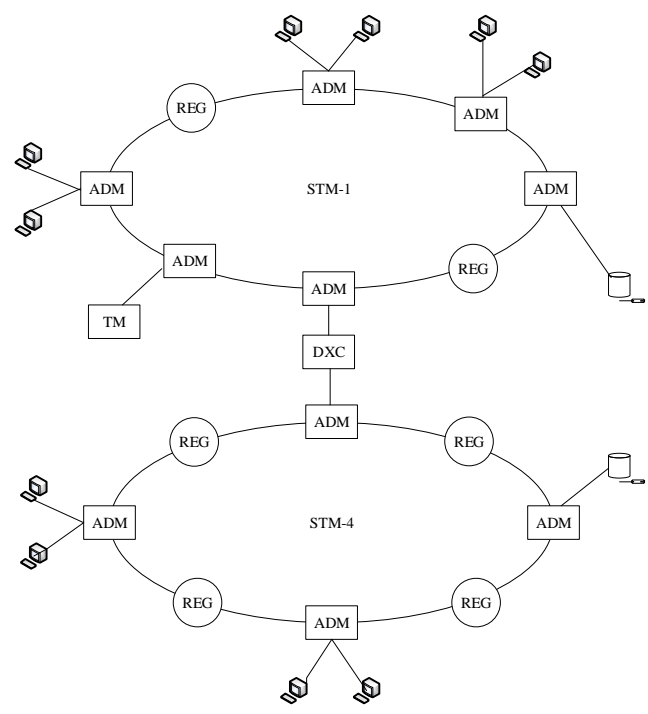

FIGURE I.BASIC NETWORK ELEMENTS IN THE SDH NETWORK

The SDH equipment simulation models are established as follows: according to the signal processing flow and the role of each device in signal processing, the functional simulation of each logic module is performed according to the signal flow processing sequence. And then, under the unified management module organization control, the multiplexing and demultiplexing of SDH signal frame can be completed. The main functions of TM, ADM, and DXC are the multiplexing , demultiplexing and digital cross-connect.

a) The realization of multiplexing function

The low-speed line signal is multiplexed to the high-speed line signal, which needs to be mapped, positioned and multiplexed in three steps. Figure 2 shows how to combine multiple services onto the STM trunk. This is the example of a E1 (2Mbit/s) service in China.

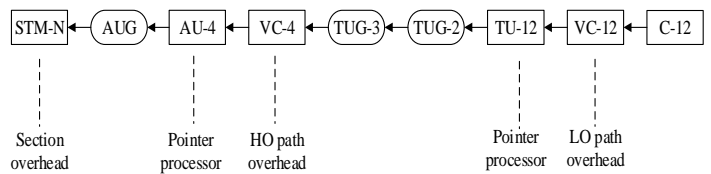

FIGURE II. MULTIPLEXING STRUCTURE IN CHINA FLOW CHART

The multiplexing process is as follows:

- The E1 frame is placed into a C-12 Container.

- A Path overhead is added and it becomes a VC2 Virtual Container.

- Multiple VC-12s are assigned Pointers and become a TUG-2 Tributary Unit Group. The pointers indicate the location of the first byte of each of the Virtual Containers.

- Seven of these TUGs can be Mapped into a VC3 Virtual Container.

- Multiple VC-3 Virtual Containers will be assigned Pointers and placed into an AUG Administration User Group.

- And the AUG will be placed in the STM Frame. The Pointers are used to locate individual 2 meg streams in the STM Frame

In order to simulate this process, this paper designs the flow chart when the program works, as shown in Figure 3.

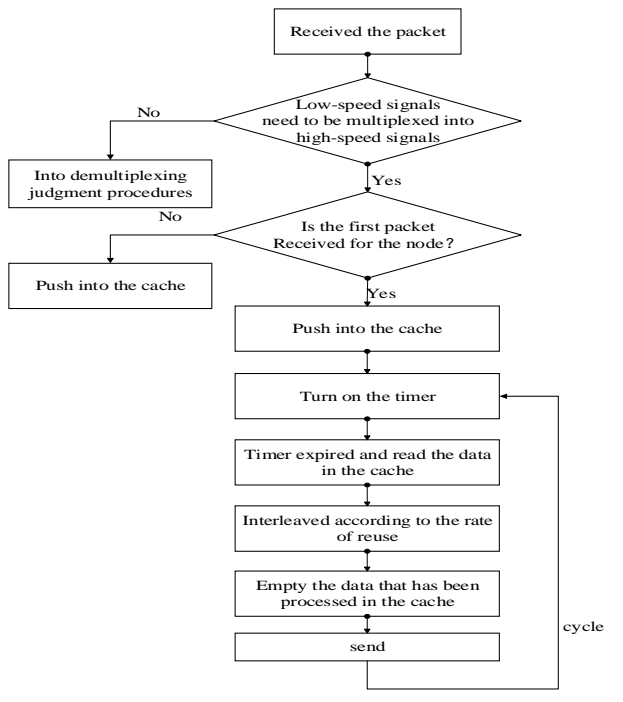

FIGURE III.

MULTIPLEXED FLOW CHART 
b) The realization of demultiplexing function

To demultiplex high-speed line signals to low-speed line signals, it is necessary to analyse the rates before and after demultiplexing and decompose the low-speed signals according to the interpolation rules when multiplexing. Demultiplexing is the inverse of multiplexing. In order to simulate this process, the low-speed signal packets sent to the next node as shown in Figure 4.

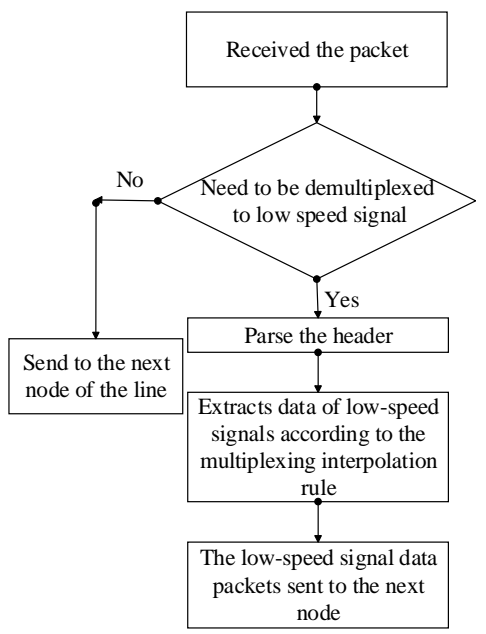

FIGURE IV.

DEMULTIPLEXING FLOW CHART

\section{c) Digital Cross Connect(DXC)}

The digital cross-connect function converts the rate of the input signal to the rate of the output port. Therefore, after receiving the data packet, it needs to determine whether it needs to perform rate conversion or not, and directly go to the multiplexing program. This function achieves the multiplexing process from STM-m to STM-n $(m<n)$. In order to simulate this process, this paper designs a flowchart of the program, as shown in Figure 5.

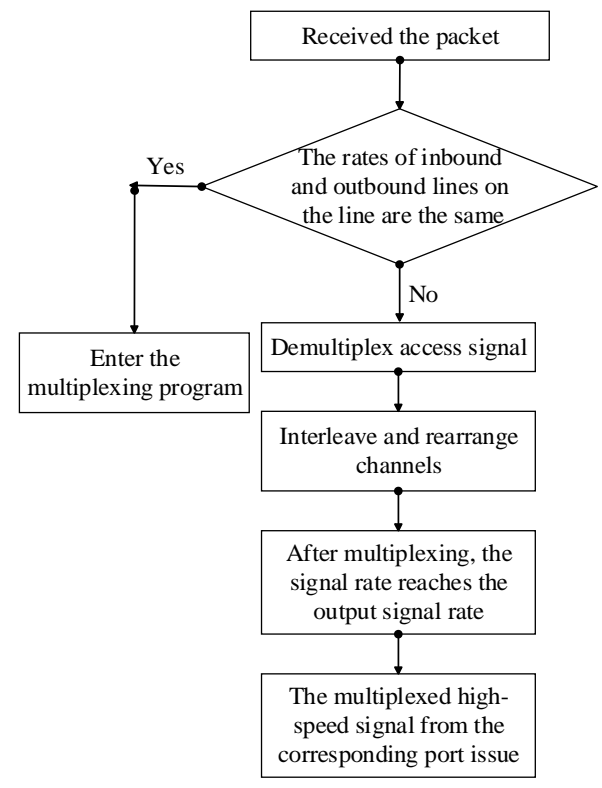

FIGURE V.

DIGITAL CROSS-CONNECT FLOW CHART

\section{d) Regenerator(REG)}

The regenerative repeater REG mainly converts the optical signal into an electrical signal, and then undergoes sampling, amplification shaping, and regeneration planning as electrical signals, and then modulates into optical pulse signal transmission to ensure the integrity of the transmitted signal waveform. At the same time, the transmission distance is also prolonged. Since REG's main functions are concentrated in the physical layer, this paper adopts transparent transmission of data packets to simulate the function of the device.

\section{2) Network layer modeling}

The network layer modeling mainly includes link, protocol, network traffic modeling and network topology modeling. The SDH transmission network is an optical fiber link, so it only needs to model the link delay. In this paper, EoS (Ethernet over $\mathrm{SDH})$ technology[13] is used to map Ethernet signals to data frames transmitted by SDH devices. The process includes the following steps:

- Delete the preamble and frame start delimiter of the MAC frame;

- The data frame is encapsulated based on the Link Access Procedure SDH (LAPS) protocol;

- The encapsulated data frame is mapped to the SDH VC (virtual container) virtual concatenation to achieve transmission;

- The receiver extracts and parses the protocol frames in the VC virtual concatenation;

- $\quad$ Add the preamble and start of frame delimiter.

The recovery of LAPS protocol frames is closely related to its mapping. At the receiving end, the recovery of LAPS protocol frames requires the following three steps:

- $\quad$ Receive data from VC-12-3v;

- The data frame is encapsulated based on the Link Access Procedure SDH (LAPS) protocol;

- $\quad$ Restore LAPS protocol frames.

Due to the size of the Ethernet and IP packet signals is not as same as the fixed size of the SDH virtual container VC, the resource utilization is low. In order to improve the resource utilization, SVC VC cascading technology is adopted, which is specifically implemented as a cascade of $\mathrm{N}$ VCn virtual containers equivalent to a container whose capacity size is $\mathrm{N} \times \mathrm{VCn}$. In practical applications, the dynamic adjustment of the packet signal capacity in the transmission process can be dynamically adjusted to improve the channel utilization of the $\mathrm{SDH}$.

The network topology structure modeling adopts an objectoriented modeling mechanism and establishes the equipment model and connection model of the power communication network according to the types of power communication networks (such as point-to-point, bus, and wireless, etc.). Starting from the type of power communication network, the network topology modeling mainly includes point-to-point, bus, 
and wireless structures. Based on the object-oriented modeling mechanism, the internal equipment model and link model of the power communication network are reasonably structured. Starting from different network functions, the model version of the complete network equipment is implemented to support various simulation needs.

For different occasions, the following three traffic modeling techniques are used to establish a network traffic simulation model.

- Explicit traffic modeling: construct a simulation process model for generating, queuing, transmitting and receiving data packets, and clarify the process traffic;

- Background interactive traffic modeling: describe endto-end traffic according to the traffic routing conditions and traffic matrix;

- Background utilization flow modeling: When there is no simulation packet transmission, this model clarifies the background traffic in the network, which is mainly used for capacity planning and application analysis.

\section{3) Application layer modeling}

Application modeling is actually about data flow modeling. This paper uses a standard application model to simulate the required services. There are two types involved in the simulation: the OFF/ON application model and the end-to-end application model.

The OFF/ON application model is a mathematical analysis model. This application is represented by the discontinuity of data transmission, and the alternating of ON and OFF states. Among them, there is data transmission in the ON state and no data transmission in the OFF state. In general, the ON state and the OFF state are subject to independent distribution. To describe the random elements of the OFF/ON data source, it is necessary to grasp the distribution of the ON and OFF interval lengths. This service model can not only set the time period for each node device to start sending packets, but also can set the packet size and the arrival interval of each packet.

The end-to-end application model based on Client/Server (client/server) is service model. Common application protocols have been set and modularized. Users can select corresponding parameters according to service requirements and configure such standard applications. It is generally divided into four steps: setting application parameters; setting application inquiries; configuring applications supported by the server and setting client service inquiries.

\section{B. Self-healing Behavior Simulation}

Self-healing behavioral simulations are mainly divided into fault occurrence behavior, fault detection behavior and selfhealing recovery behavior.

\section{1) Fault occurrence behavior modeling}

The common faults in the SDH network fall into three categories: transmission equipment faults, link faultsand peripheral equipment faults.

\section{a) Transmission equipment faults}

Transmission equipment faultshappens in the emulation device. All ports or fault ports of the equipment are set to be unavailable, and the fault information is synchronized to the adjacent node ports of the faulty port. That is to say, the node fault makes all timeslots unavailable of the adjacent node ports .

\section{b) Link faults}

Link faults happens in the emulated device, the connected device port is set to the unavailable state. That is to say, the link fault makes the ports of both ends of the link unavailable.

\section{c) Peripheral equipment faults}

Peripheral equipment [14] such as synchrophasor measurement devices and stability control devices are greatly affected by the external environment. Maintenance personnel are required to pre-qualify the power supply on a regular basis to ensure stable, safe, and stable operation of the equipment. Such faults require human intervention, so they areunable to recover business through self-healing rings.

\section{2) Fault detection behavior}

The fault detection of the SDH network is divided into two modes: fault detection of the common transmission equipment and the selective equipment.

\section{a) Fault detection of common transmission equipment}

The ordinary transmission device detects the status of each time slot on each sending port per $0.125 \mathrm{~ms}$, including whether the message received by the time slot carries alarm information and whether the receiving port of the time slot is available.

If there is an alarm information in the current time slot or the receiving port of the time slot is unavailable, a message carrying the alarm information is sent; if the fault has no effect on the current time slot of the current node, a normal message is sent.

\section{b) Fault detection of the receiving equipment}

The receiving device receives the same message from the primary slot and the backup slot. Normally, the message from the primary path does not carry alarm information, and the receiving device receives the message from the primary path.

When the message of the primary path carries the alarm information of a certain time slot, the selective receiving node detects that the primary path of the time slot is faulty and takes corresponding protective measures. If the selective receiving device only receives the message of the backup path, the corresponding protection measure is adopted in the case of confirming the failure of the active path.

\section{3) Self-healing recovery behavior modeling}

In the communication and transmission process of power grids, there are many self-healing protection methods for data and information communication transmission in SDH networks. According to the functional structure of actual power communication networks, the self-healing protection method chosen in this paper is automatic line switching protection [15]. 

ways:

According to the actual use requirements, there are two

\section{a) Automatic does not return}

The receiving node works on the main line. If the main line is faulty, the receiving node automatically selects the standby line message and works on the standby line (except for manual intervention or failure of the standby line); after that, until the backup line fails, select The receiving node is automatically switched to the main line (except for manual intervention or failure of the main line).

\section{b) Automatic return}

The receiving node works on the main line. If the main line fails, the receiving node automatically selects the standby line message and works on the standby line; after that, if the main line returns to normal and can operate normally for a period of time, the receiving node automatically Return to the main line.

\section{SimULATION EXAMPLE AND RESUlT}

\section{A. Self-healing Process Example}

\section{1) Simulation scene}

Based on the topology of the SDH communication network in Leshan Sichuan Province, this paper implements self-healing simulation of the power SDH transmission network. The network includes an access layer ring network and a corresponding collecting layer ring network. The service is a security control and real-time data transmission service. The service bandwidth of each channel is generally $2 \mathrm{Mbps}$ and the link channel adopts two-fiber bidirectional channel protection. The receiving equipments include a PMU (power management unit) device and a power stability control device, the frequencies of the two packages are $0.01 \mathrm{~s}$ and $0.833 \mathrm{~ms}$ respectively.

Under normal circumstances, the SDH network adopts a dual-transmission protection mechanism. After the terminal equipment information is multiplexed by the SDH equipment, it is transmitted at the same time in the active path and the backup path, the final selection node on the ring chooses to receive messages from the primary path with better communication quality. The network simulation scenario is shown in Figure 6.

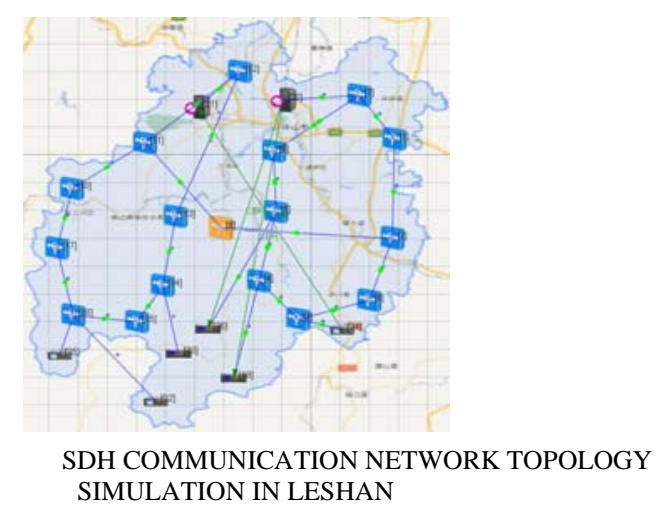

\section{2) Fault behavior simulation}

The SDH network fault simulation includes two types: communication device fault and link fault. The communication device fault is a red flag and the link failure is a yellow flag as shown in Figure 7.

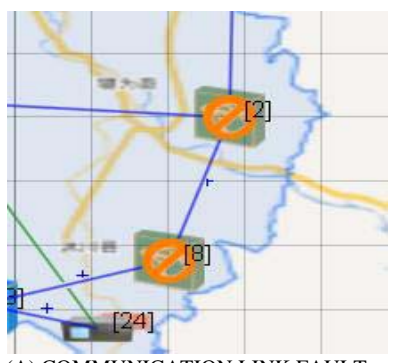

(A) COMMUNICATION LINK FAULT FIGURE VII.

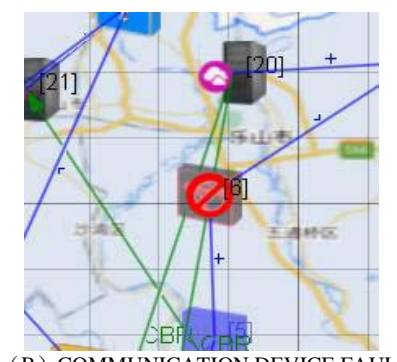

(B) COMMUNICATION DEVICE FAULT

The total simulation time is 1 hour. The fault includes:

Starting from $1 \mathrm{~min}$, once per $40 \mathrm{~s}$, a link fault occurs for a duration of 20s, affecting the primary path of one time slot of the same ring and the backup path of two time slots;

Starting from $2 \mathrm{~min}$, once per $1 \mathrm{~min}$, there is a communication device fault for a duration of $20 \mathrm{~s}$, affecting the primary path of two time slots of the same ring and the backup path of one time slot.

\section{3) Self-healing recovery simulation}

There are two ways to recover from self-healing: automatic with no return and automatic with return, you can set the recovery mode of self-healing recovery nodes in the graphical interface, as shown in the figure below.

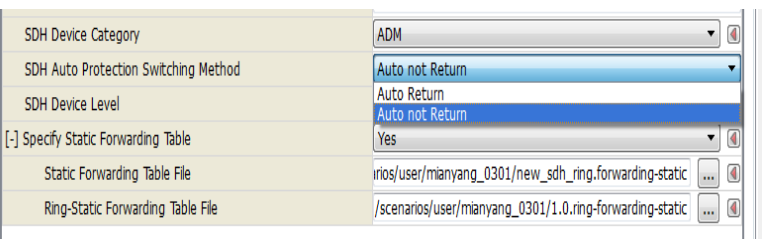

FIGURE VIII.
SELF-HEALING RECOVERY MODE SETTING

In the automatic with return mode, the self-healing process is:

- 1 min-1min20s, node 2 is as the selection node of the time slot. Then link is switched to the backup path, and always work in the backup path;

- 2 min-2min20s, the primary path of node 21 and node 24 is affected by the fault, it switches to the backup path, and keeps always working on the backup path .If the backup path of node 2 is faulty, it switches back to the primary path, and keeps working on the primary path.

- 2 min40s-3min, the backup paths of node 21 and 24 are affected by the fault, switch back to the primary path, and always keep working in the primary path; the primary path of node 2 is faulted, switches to the 
backup path, and always keeps working in the backup path.

In the automatic with no return mode, the self-healing process is:

- 1 min-1min20s, node 2 as the selection node of the time slot, switches to the backup path, and has been working in the backup path;

- 1 min21s, node 2 receives a valid message from the active path for this time slot, switches back to the primary path, and works in the primary path.

- $\quad 2$ min-2min20s, the primary paths of node 21,24 , and 2 are affected by the fault, switching to the backup path, and always work in the backup path.

- 2 min21s, the node 21,24 , and 2 receive a valid message from the primary path in the time slot, switch back to the primary path, and work in the active path.

The subsequent self-healing process repeats the above four processes.
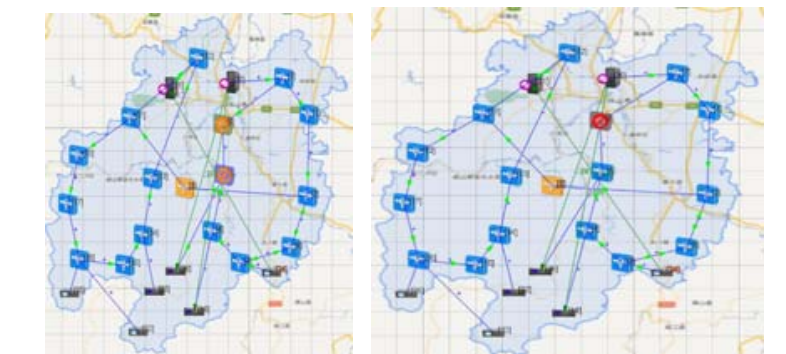

(A) SELF-HEALING LINK FAULT (B) SELF-HEALING COMMUNICATION EQUIPMENT FAUL

FIGURE IX.

SDH NETWORK SELF-HEALING SIMULATION

Comparing the statistical results of sending and receiving services, you can find that based on the self-healing model of this paper, the fault of the SDH network and its recovery process are simulated, and there is no packet loss phenomenon.

\section{B. Self-healing Ring Application Evaluation Example}

Figure 10 shows a protected communication network in a certain area. The network includes 2 provincial transmissions, 2 tones, 16 substations, and 2 power plants. The flow of its business is mainly for each $500 \mathrm{kV}$ substation and the ground adjustment sub-power plants and $220 \mathrm{kV}$ substations.

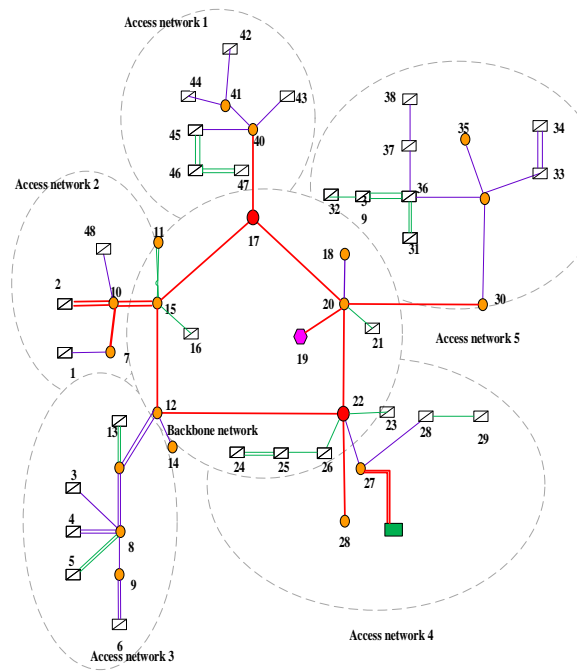

FIGURE $X . \quad$ TOPOLOGY OF A PROTECTED COMMUNICATIONS NETWORK IN AN AREA

The application will be brought to the province to adjust, while provincial regulation, tones, substations issued corresponding protection orders. In order to increase the reliability of the network, each station node of the plant uses a dual 2M fiber link to uplink to the backbone node through the router. After the links are bundled, the backbone node is connected to the ground adjustment point of its own area.

The protection communication special network is divided into six areas, including five star-access networks and a ring backbone network. Stations 19 is provincial deployment site and the collecting core of the entire system protection communication network. Station 15, station 17, station 20 and station 22 are collecting cores of access network 1, access network 2, access network 3, network 4 and network 5, respectively, and station 12 , station 15 , station 17 , station 20 and station 22 are connected through a fiber ring network. The backbone network forms the system protection communication network, and each access network is connected to each site in a star manner.

In an actual network, because the failure rates of network elements are not the same, the failure rate of each network element can be obtained by counting the failure-free working time of each element of the network for a period of time. According to the actual network data, the failure rate of each network element is entered in the simulation model, and the availability parameter of the system protection communication network is obtained. 
TABLE I. AVAILABILITY OF SYSTEM PROTECTION COMMUNICATION

\begin{tabular}{|c|c|c|c|c|c|}
\hline $\begin{array}{c}\text { Network } \\
\text { name }\end{array}$ & $\begin{array}{c}\text { Number } \\
\text { of } \\
\text { interfaces }\end{array}$ & $\begin{array}{c}\text { Device } \\
\text { interface } \\
\text { availability }\end{array}$ & $\begin{array}{c}\text { Fiber } \\
\text { count }\end{array}$ & $\begin{array}{c}\text { Optical } \\
\text { fiber } \\
\text { availability }\end{array}$ & $\begin{array}{c}\text { Overall } \\
\text { availability }\end{array}$ \\
\hline $\begin{array}{c}\text { Overall } \\
\text { network }\end{array}$ & 160 & 0.999879 & 75 & 0.999968 & 0.999759 \\
\hline $\begin{array}{c}\text { Backbone } \\
\text { network }\end{array}$ & 20 & 0.999716 & 10 & 0.999889 & 0.999714 \\
\hline $\begin{array}{c}\text { Access } \\
\text { Network 1 }\end{array}$ & 22 & 0.999759 & 10 & 0.999889 & 0.999741 \\
\hline $\begin{array}{c}\text { Access } \\
\text { Network 2 }\end{array}$ & 20 & 0.999716 & 9 & 0.999836 & 0.999695 \\
\hline $\begin{array}{c}\text { Access } \\
\text { Network 3 }\end{array}$ & 32 & 0.999685 & 15 & 0.999806 & 0.999654 \\
\hline $\begin{array}{c}\text { Access } \\
\text { Network 4 }\end{array}$ & 28 & 0.999762 & 13 & 0.999854 & 0.999738 \\
\hline $\begin{array}{c}\text { Access } \\
\text { Network 5 }\end{array}$ & 38 & 0.999683 & 18 & 0.999926 & 0.999814 \\
\hline
\end{tabular}

From Table I, it can be seen that the fiber optics availability and overall availability of the backbone network are significantly higher than the fiber access and overall availability of the access network, which is due to the channel protection function of the SDH self-healing ring and the effect of fiber failure on the reliability of the entire network. It can be seen that compared with a star network, the reliability of the ring network structure is better, and it is more suitable for the backbone layer and the core layer. At the same time, improving the availability of device ports is the key to improving the overall network reliability.

\section{CONCLUSION}

In view of the current situation of research on the selfhealing ability assessment of the system protection network, this paper first models the simulation resources of equipment, networks, and services in the SDH optical communication transmission network. And then, when the faults occur in the network, the behavior of the SDH equipment and the state change process are simulated, so the full-process simulation of the self-healing function can be realized in the SDH transmission network. Additionally, the whole process simulation was performed to intuitively demonstrate the selfhealing capability and effects in the SDH network. Finally, the availability of the SDH optical communication transmission network are evaluated by statistical network effectiveness, and it is verified that the channel protection function of the SDH self-healing ring can improve the security and reliability of the grid.

\section{ACKNOWLEDGMENT}

This paper is supported by the State Grid Technical project: "Research on Communication Architecture and Hardware-InThe-Loop Simulation for Real-Time Wide-Area Stability Control of Electric Power System (5442XX160008-XX71-16002)”.

\section{REFERENCES}

[1] Wang Hua, Xiao Bing, Zhao Yuliang, et al. Study on Transmission Nodes Number, Time Delay and Protection Mode of SDH Optical Fiber [J]. Qinghai Electric Power, 2009, 28(3): 7-10.

[2] Wang Xiaqing. Talking about the Error of SDH Optical Fiber Transmission System[J]. Science \& Technology Information, 2011(6):246-246.

[3] XJ Liang. Talk on the Application of SDH Self-healing Protection in Province Transmission Network[J], Mechanical Management \& Development, 2012, (1), 206-209.

[4] WJ Yin, D Yuan, JG Li, RY Han, etc. Research on wide-area protection system based on SDH network[J]. Power System Protection \& Control, 2011, 39 (5) :120-122.

[5] Li Yang, Ma Wei, Gu Xingmin. Application of SDH optical fiber selfhealing protection channel in power communication network [J]. 2011, (1): 39-42.

[6] ZH Cui, LI Lin-Chuan, CS Feng, B Chen. Research of SDH SelfHealing Mechanism for Line Current Differential Protection[J]. Telecommunications for Electric Power System, 2012, 33(240): 44-48.

[7] Bai Jingru, The Design and Improvement of SDH Self-healing Ring [D], Beijing University of Posts and Telecommunications, 2009.

[8] Clarkson T, Clarkson T. Simulation tools for multilayer fault restoration[M]. IEEE Press, 2009.

[9] Calvet J T, Girault C. A Simulation Environment for SDH Synchronization Network Planning[J]. 2001.

[10] Schwartz S H, Zager D. Value-oriented network management[C]// Network Operations and Management Symposium, 2000. NOMS 2000.2000 IEEE/IFIP. IEEE, 2000:715-728.

[11] Ruepp S, Dittman L, Ellegard L. Simulation and comparison of path restoration techniques in SDH mesh networks[C]// Drcn 2005). Proceedings. International Workshop on Design of Reliable Communication Networks. IEEE, 2005:47-53.

[12] Li Jianhua, Zhang Ligang, Wang Rong, et al. Design of the SDH Equipment Training Simulator Based on Client /Server Mode [J]. Military Communications Technology, 2001(4):41-44.

[13] Chen Shoulong, Ge Taixiang. Application Comparison of ATM Technology and EOS Technology[J]. Electric Power System Communication, 2010, 31(12):45-47.

[14] Cheng Guangming, Liao Minghong, Wu Xianghu. Management of onboard computer and its peripherals [J]. Journal of Harbin Institute of Technology, 2002, 34(2): 201-203.

[15] Li Yang, Ma Wei, Gu Xingmin. Application of SDH optical fiber selfhealing protection channel in power communication network [J]. 2011, (1): 39-42. 